\title{
Stochastic 3D Modeling of Non-Woven Materials with Wet-Proofing Agent
}

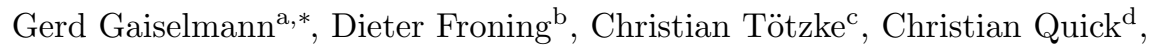 \\ Ingo Manke ${ }^{\mathrm{c}}$, Werner Lehnert ${ }^{\mathrm{b}, \mathrm{e}}$, Volker Schmidt ${ }^{\mathrm{a}}$ \\ ${ }^{a}$ Institute of Stochastics, Ulm University, Germany \\ ${ }^{b}$ Institute of Energy and Climate Research (IEK-3: Electrochemical Process Engineering), \\ Forschungszentrum Jülich GmbH, Germany \\ ${ }^{c}$ Institute of Applied Materials, Helmholtz Centre Berlin for Materials and Energy (HZB), \\ Germany \\ ${ }^{d}$ Fa. Freudenberg Fuel Cell Components Technology KG (FFCCT), Germany \\ ${ }^{e}$ Modeling in Electrochemical Process Engineering, RWTH Aachen University, Germany
}

\begin{abstract}
A novel, realistic 3D model is developed describing the microstructure of nonwoven GDL in PEMFC which consists of strongly curved and non-overlapping fibers. The model is constructed by a two-stage procedure. First we introduce a system of random fibers, where the locations of their midpoints are modeled by a 3D Poisson point process and the fibers themselves by random 3D polygonal tracks which represent single fibers in terms of multivariate time series. Secondly, we transform the random fiber system into a system of non-overlapping fibers using an iterative method leaned on the so-called force-biased algorithm. The model is validated by comparing transport-relevant characteristics computed for experimental 3D synchrotron data, and for realizations sampled from the stochastic microstructure model. Finally, we suggest a model for the spatial distribution of PTFE, a wet-proofing agent often used in non-woven GDL, and combine this PTFE model with our new microstructure model for non-woven GDL.
\end{abstract}

Keywords: fiber-based material, stochastic modeling, vectorial autoregression, non-woven, PTFE, synchrotron tomography

\section{Introduction}

Proton exchange membrane fuel cells (PEMFC) are a seminal technology for the generation of electrical power from hydrogen being efficient and eco-friendly. A key component of PEMFC is the gas-diffusion layer (GDL), whose main functions are the gas supply of the electrodes, the regulation of water storage, *Corresponding author: phone: +4973150 23590, fax: +49 73150 23649, e-mail:
gerd.gaiselmann@uni-ulm.de

Preprint submitted to Elsevier

April 23, 2013 
and evacuation within the GDL. The microstructure of non-woven GDL consists of highly curved fibers. Their morphology strongly determines the efficiency of the fuel cell as well as its stability $[11,14,17]$. Thus, the optimization of the GDL's microstructure is of utmost importance.

However, even for a known 3D microstructure of GDL material, it remains a challenging task to quantitatively predict its physical properties. In practice, a suitable microstructure for the GDL is usually determined by experiments based on trial-and-error. A more sophisticated way for the design of GDL microstructures is to generate synthetic ones using validated stochastic models. Then, by means of model-based computer experiments, microstructures can be detected such that the physical processes are improved. More precisely, by varying the values of the model parameters in a systematic manner, a large variety of different microstructures can be simulated. Subsequently, the goodness of these microstructures can be evaluated by numerical (transport) processes computed on the synthetic microstructures. Thereby, 3D microstructures with enhanced transport properties can be identified.

In this paper, we present a novel, realistic 3D model describing the microstructure of non-woven GDL in PEMFC which consist of strongly curved and non-overlapping fibers. There exist several stochastic models of fiber-based materials in literature, where in $[2,4,5,6,13,20,22,23,24]$ models are considered which are designed for fiber-based materials consisting of straight fibers. In contrast to these approaches, in $[1,10,19]$ stochastic models are proposed which describe materials consisting of curved fibers.

The modeling approach introduced in [1] is based on simulating a chain of spheres where the sphere midpoints follow a random walk. Thereby, each sphere chain is assigned a main direction according to a beta-orientation distribution. Given the preceding and the main direction of the sphere chain, the next direction is drawn from a multivariate von-Mises-Fisher distribution. In this way, curved fibers can be simulated using a small number of parameters. Moreover, this modeling approach offers a nice control of the directional distribution of the fibers. In particular, the main direction of a single fiber can be adjusted. However, when it comes to modeling fibers which exhibit extremely large curvature as loops, for example, other modeling approaches appear more suitable. In [10], a multi-layer model for curved, but horizontally orientated, overlapping fibers is introduced, where $2 \mathrm{D}$ random polygonal tracks representing planar fibers are simulated according to multivariate time series. Thereby, for instance, it is possible to simulate fibers exhibiting repeating loops, see also Figure 1, by adjusting the parameters of the time series adequately. The benefit of the times-series approach is that the correlation of consecutive line segments can be taken into account. This is a great advantage for modeling the local course of fibers. Note, however, that the assumption of solely horizontally orientated fibers, is rather restrictive and not fulfilled for many fiber-based GDL materials.

In [19], another multi-layer model for systems of non-overlapping fibers is introduced. This modeling approach seems to be suitable to describe the structure of woven fiber materials. Note, however, that in the example of application considered in the present paper, we aim to describe non-woven GDL, which 


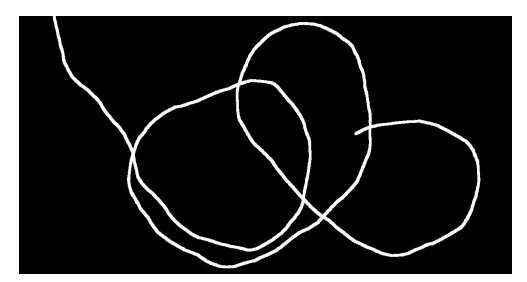

Figure 1: Planar fiber generated by the random polygonal track model based on multivariate time series

exhibit strongly curved fibers.

In the present paper, we introduce a novel model that describes non-overlapping fibers with strong curvatures and apply this model to a non-woven GDL material consisting of carbon fibers. Thereby, a fiber model is used which is an alternative to the model proposed in [1]. In particular, the fibers drawn from our model can exhibit arbitrarily large curvatures (e.g. loops) in contrast to those obtained by the model introduced in [1]. But, on the other hand, the directional distribution of fibers obtained by our model can not be controlled in such an efficient way as in [1]. The fiber model considered in the present paper is a generalization of the multi-layer approach given in [10]. More precisely, fibers are now directly modeled in 3D (i.e., not by a multi-layer approach) using a single 3D time series. Furthermore, in the microstructure model proposed in the present paper, an avoidance algorithm is incorporated which prevents the fibers from mutual penetrations. Thus, the proposed model can describe fiber-based materials more realistically than the model considered in [10].

In addition, our model allows the consideration of super-structures, e.g. clusters of fibers, which are observed in real non-woven GDL materials. Finally, since non-woven GDL are often treated with polytetrafluoroethylene (PTFE) (to increase hydrophobicity and to reduce blocked pathways caused by water holdups), we incorporate a wet-proofing agent (PTFE) into the model.

The stochastic microstructure model proposed in the present paper is constructed by means of methods from stochastic geometry and multivariate time series analysis (see e.g. $[12,18,21]$ and $[7,16]$, respectively). In particular, a two-stage approach is used, where we first consider a germ-grain model to generate a system of overlapping fibers. The germs form a homogeneous Poisson point process in $3 \mathrm{D}$ and the grains are random $3 \mathrm{D}$ polygons described by a suitable multivariate time series, which are spherically dilated in 3D. Subsequently, the germ-grain model is transformed in the following way: First the fibers are translated such that they are evenly spread in space. Secondly, like in [1], an iterative avoidance algorithm is applied to the translated fiber system to eliminate overlaps between the fibers. This model is then used to suitably describe the microstructure of non-woven GDL in PEMFC. More precisely, the parameters of the non-woven GDL model are fitted to 3D image data of experimental non-woven GDL, where the image data is gained by synchrotron tomography. To adequately fit the model parameters, we extract a system of single fibers 
from experimental image data of non-woven GDL using an algorithm described in [9]. From the extracted fiber system, important properties like curvature measures or directional distributions of the fibers can be incorporated into the model. Finally, for model validation, transport-relevant characteristics computed for experimental data of non-woven GDL and those computed for virtual non-woven GDL drawn from the fitted model are compared to each other, where a good agreement is found.

The paper is organized as follows. Section 2 briefly describes an algorithm for the detection of single fibers in 3D synchrotron images. In Section 3, the stochastic model for 3D polygonal tracks is introduced. Section 4 establishes the 3D model for non-woven GDL. In Section 5 it is shown how PTFE can be included into the model as a further material component. Section 6 concludes the obtained results.

\section{Automated detection of fiber courses}

In this section, we briefly describe an algorithm to automatically extract systems of single fibers from 3D tomographic data of fiber-based materials. This algorithm combines tools from image processing and stochastic optimization. It is described in detail in [9] and thus we only mention the basic idea.

In a first processing step the 3D image is binarized (by global thresholding). Then we focus on the extraction of center lines of single fibers from the binarized image. Due to irregularities like noise or binarization artefacts it is only possible to extract relatively short fragments of the center lines. Subsequently a stochastic algorithm is considered which accurately connects these parts of the center lines to each other, in order to reconstruct the complete fibers such that the curvature properties of the fibers are represented correctly.

Exemplarily, we apply this algorithm to 3D image data of non-woven GDL, gained by synchrotron tomography. In Figure 2 the result which has been obtained by our detection algorithm is displayed. The experimental data and the system of fibers extracted from it are in good optical accordance. In [9], it is shown more formally that the algorithm extracts fibers accurately with respect to curvature characteristics. In particular, for a representative test volume of fiber-based material, the algorithm reproduced $91 \%$ of connections correctly. The extracted system of single fibers is the data basis for the parameter fitting of the single-fiber model introduced in Section 3 below. Note that the extracted fibers are represented by polygonal tracks.

\section{Modeling of single fibers and fiber bundles}

We introduce a stochastic model, which extends the modeling approach considered in [10]. It describes the typical course of those fibers that have been extracted from 3D synchrotron data using the extraction algorithm described in Section 2. Note that the extracted fibers are available as polygonal tracks. Thus, our suggestion for modeling the course of strongly curved fibers is a random polygonal track which is based on multivariate time series. 

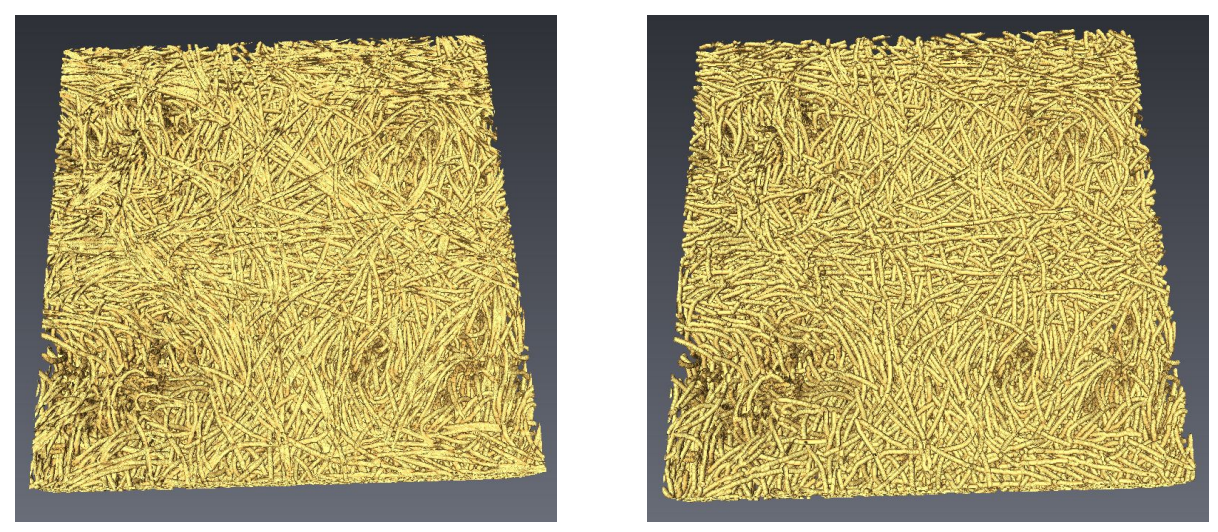

Figure 2: Experimental data gained by synchrotron tomography (left) and extracted fibers (right)

\subsection{Incremental representation of polygonal tracks}

The following incremental representation of polygonal tracks is useful. Instead of describing a polygonal track $p=\left(p_{0}, p_{1}, \ldots, p_{n}\right)$ by the endpoints $p_{i}, p_{i+1} \in \mathbb{R}^{3}$ of its line segments $\left(p_{i}, p_{i+1}\right)$ we consider an angle-length representation, where we regard the first line segment $\left(p_{0}, p_{1}\right)$, separately. The further segments of the polygonal track $p$ can then be described by the lengths $\ell_{1}, \ell_{2}, \ldots$ of the consecutive line segments and the angles $\alpha_{1}, \alpha_{2}, \ldots$ and $\beta_{1}, \beta_{2}, \ldots$, where $\alpha_{i}\left(\beta_{i}\right)$ denotes the change of direction from the $i$-th to the $(i+1)$-th segment with respect to the azimuthal (polar) angle. Thus, under the condition that the first line segment is given, a polygonal track is uniquely described by the sequence of vectors $\left(\alpha_{1}, \beta_{1}, l_{1}\right)^{\top},\left(\alpha_{2}, \beta_{2}, l_{2}\right)^{\top}, \ldots$, see Figure 3 .

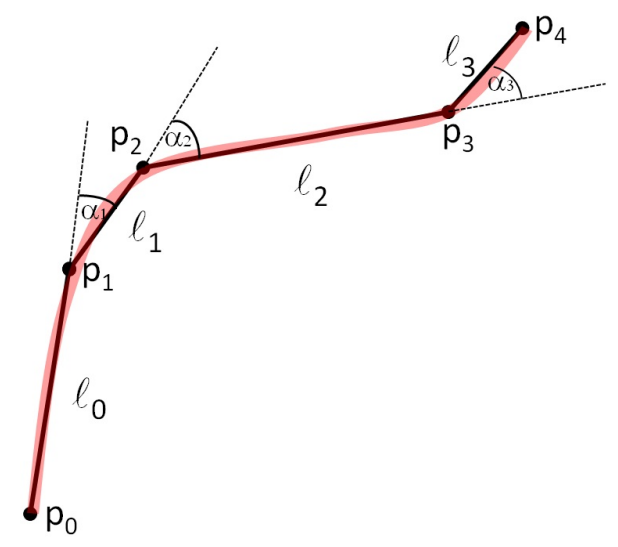

Figure 3: Planar fiber (red) represented by a 2D polygonal track 


\subsection{Model description}

Based on the incremental representation of polygonal tracks given in Section 3.1 we first introduce the single-fiber model. The main idea for stochastic modeling of single fibers is to describe polygonal tracks (representing the fibers) by a three-dimensional time series $\left\{F_{i}, i \geq 1\right\}$. The geometric interpretation of $\left\{F_{i}, i \geq 1\right\}$ is that the first (second) components of the random vectors $F_{i}=\left(F_{i 1}, F_{i 2}, F_{i 3}\right)$ form a sequence of random angles whose realizations are denoted by $\alpha_{1}, \alpha_{2}, \ldots\left(\beta_{1}, \beta_{2}, \ldots\right)$, where $\alpha_{i}\left(\beta_{i}\right)$ is the change of direction from the $i$-th to the $(i+1)$-th line segment with respect to the azimuthal (polar) angle. The third components $F_{i 3}$ specify the random lengths of the consecutive line segments of a random polygonal track whose realizations are denoted by $\ell_{1}, \ell_{2}, \ldots$. By the usage of multivariate time series we are in a position to include cross-correlations of angles and lengths of line segments which are close together into the model. This is essential in order to properly reflect the geometrical properties of the extracted fibers by our model. In particular, we are able to describe fibers with arbitrarily strong curvatures.

Thus, we consider the following modeling components $F_{0},\left\{F_{i}, i \geq 1\right\}$ to build the stochastic single-fiber model which represents a random polygonal track with initial line segment given by $F_{0}=\left(F_{01}, F_{02}, F_{03}\right)$ and total length of all line segments equal to $\ell$, where each line segment is dilated by a $3 \mathrm{D}$ sphere $b(o, r)$ with center at the origin and radius $r$. Note that the parameter $r$ represents the radius of the fibers. In other words, the single-fiber model $X=\left\{X_{0}, \ldots, X_{N}\right\}$ with $N=\min \left\{k: F_{0}+\sum_{i=1}^{k} F_{i 3}>\ell\right\}$ is considered, where its dilated line segments $X_{i}$ are given by

$$
X_{i}= \begin{cases}\left(o, L_{1}\right) \oplus b(o, r), & \text { if } i=0, \\ \left(L_{i}, L_{i+1}\right) \oplus b(o, r), & \text { else },\end{cases}
$$

with $L_{1}=T\left(F_{0}\right), L_{i+1}=L_{i}+T\left(\sum_{j=0}^{i} F_{j 1}, \sum_{j=0}^{i} F_{j 2}, F_{i 3}\right)$ for $i \geq 1$ and $T(\phi, \theta, s)=(s \sin (\theta) \cos (\phi), s \sin (\theta) \sin (\phi), s \cos (\theta))^{\top}$ being the transformation from spherical to Euclidean coordinates.

We assume that the time series $\left\{F_{i}, i \geq 1\right\}$ is stationary, i.e., the mean and covariance of the time series do not change over time. Thus, it can be extended to a stationary time series $\left\{F_{i}, i \in \mathbb{Z}\right\}$, where $\mathbb{Z}=\{\ldots,-1,0,1, \ldots\}$ denotes the set of all integers. Then, for the modeling of random polygonal tracks, we represent $\left\{F_{i}, i \in \mathbb{Z}\right\}$ by a stationary autoregressive process $\left\{Y_{i}, i \in \mathbb{Z}\right\}$ such that

$$
F_{i}=\Psi\left(Y_{i}\right) \quad \text { for each } i \in \mathbb{Z},
$$

where the function $\Psi: \mathbb{R}^{3} \rightarrow[-\pi, \pi)^{2} \times \mathbb{R}$ is given by

$$
\Psi(r, s, t)=\left(r-2 k_{1} \pi, s-2 k_{2} \pi, \max \{0, t\}\right)
$$

if $\left(2 k_{1}-1\right) \pi \leq r<\left(2 k_{1}+1\right) \pi$ and $\left(2 k_{2}-1\right) \pi \leq s<\left(2 k_{2}+1\right) \pi$ for some $k_{1}, k_{2} \in \mathbb{Z}$. The definition of $\Psi$ assures some natural regularity properties, i.e., the changes of directions of consecutive line segments have to be in the interval $[-\pi, \pi)$ and the lengths of line segments have to be non-negative. 
Thus, an important modeling component for random polygonal tracks (singlefiber model) are stationary autoregressive processes $\left\{Y_{i}, i \in \mathbb{Z}\right\}$ of some order $q \geq 0$, where

$$
Y_{i}=\eta+A_{1} Y_{i-1}+\ldots+A_{q} Y_{i-q}+\varepsilon_{i} \quad \text { for each } i \in \mathbb{Z} .
$$

The 'errors' $\left\{\varepsilon_{i}, i \in \mathbb{Z}\right\}$ form a sequence of $3 \mathrm{D}$ random vectors which are independent and Gaussian with mean vector $\mathbb{E} \varepsilon_{i}=o$ and some (non-singular) covariance matrix $\Sigma=\mathbb{E}\left(\varepsilon_{i} \varepsilon_{i}^{\top}\right)$, see e.g. [7, 16]. Note that the autoregressive model is uniquely determined by the parameters $q>0, \eta \in \mathbb{R}^{3}$, the coefficient matrices $A_{1}, \ldots, A_{q} \in \mathbb{R}^{3 \times 3}$ and the covariance matrix $\Sigma$.

The single-fiber model $X=\left\{X_{0}, \ldots, X_{N}\right\}$ described above is the basis for the fiber-bundle model, where a fiber-bundle $B$ consists of a set of parallel single fibers. It is defined by

$$
B=\bigcup_{k=0}^{M}\left(X+\delta_{k}\right),
$$

where $M$ indicates the random number of parallel fibers with respect to $X$ which is assumed to have a Poisson distribution with some parameter $\kappa$. Furthermore, we assume that $\delta_{0}=(0,0,0)^{\top}$ and that the distribution of the random vector $\left(\delta_{1}, \ldots, \delta_{M}\right)$ is constructed by considering independent and uniformly distributed random vectors $\delta_{1}, \delta_{2}, \ldots$ on the boundary of the disc with midpoint $o$ and radius $2 r$ under the condition that

$$
\min \left\{\delta_{i}-\delta_{j}: i, j \in\{1, \ldots, M\}, i \neq j\right\} \geq 2 r,
$$

where the disc is orthogonally orientated with respect to the first line segment $X_{0}$ of the single fiber $X$. Note that the condition considered in (5) can be fulfilled with high probability provided that $\kappa$ is small. It ensures that fibers within bundles do not overlap. If it is not possible to sample $\delta_{j}$ for any $j \in\{1, \ldots, M\}$

such that there occurs no overlapping within the fiber bundle, the radius of the disc is iteratively increased until all $M$ fibers can be placed without overlapping.

\subsection{Model fitting}

We now shortly explain how the parameters of the autoregressive process $\left\{Y_{i}, i \in \mathbb{Z}\right\}$ (being the main modeling component for random polygonal tracks), which has been introduced in (3), can be fitted to the extracted polygonal tracks of the experimental non-woven GDL data, see Section 2. Therefore, we first transform the polygonal tracks to the incremental representation, see Section 3.1. Then, the parameters of $\left\{Y_{i}, i \in \mathbb{Z}\right\}$ are estimated by the maximumlikelihood technique, introduced in [10] and further analyzed in [8]. Following the AIC criterion, which is widely applied to estimate the order $q$ of autoregres- 
sive processes, see also [8], we obtain $q=2$ which yields

$$
\begin{array}{r}
\eta=\left(\begin{array}{c}
0.003 \\
0.0008 \\
23.5
\end{array}\right), A_{1}=\left(\begin{array}{ccc}
0.214 & 0.061 & -0.0002 \\
-0.00004 & -0.091 & 0.00002 \\
-0.53 & 1.569 & 0.114
\end{array}\right), \\
A_{2}=\left(\begin{array}{ccc}
0.106 & 0.039 & 0.0002 \\
-0.001 & -0.11 & -0.00005 \\
-0.321 & 3.846 & 0.025
\end{array}\right) \text { and } \Sigma=\left(\begin{array}{ccc}
0.08 & 0.00007 & -0.02 \\
0.00007 & 0.0018 & -0.016 \\
-0.02 & -0.016 & 207
\end{array}\right) .
\end{array}
$$

Note that the significant degree of cross-correlations expressed by the nondiagonal entries of $A_{1}, A_{2}$ and $\Sigma$ justifies the necessity to use multivariate (i.e., multi-dimensional) time series instead of univariate (i.e., one-dimensional) ones. We also remark that the diagonal entries in the matrices $A_{1}$ and $A_{2}$ describe the dependency between the individual components (length, azimuth angle, polar angle) and the corresponding components of the previous two line segments, whereas the non-diagonal entries indicate dependencies between the individual components of the current line segment and other components of previous line segments, e.g. $A_{1}(1,2)$ describes the dependency between the azimuthal angle of the current line segment and the polar angle of the previous line segment.

Moreover, the fitted values of $\left\{Y_{i}, i \in \mathbb{Z}\right\}$ are given with respect to the voxel size of the experimental $3 \mathrm{D}$ synchrotron data which is equal to $0.83 \mu \mathrm{m}$. A transformation of this voxel size can be easily obtained by multiplying all $Y_{i 3}$ with $\frac{x}{0.83}$, where $x$ is the desired new voxel size. The remaining components $F_{0}, r$ and $\ell$ of the single-fiber model are determined in the following way. From the production process of the non-woven GDL considered in this paper it is known that $r=4.75 \mu \mathrm{m}$ and $\ell=50,000 \mu \mathrm{m}$. The initial line segment given by $F_{0}$ is chosen such that the resulting directional distribution considering all line segments matches its experimental counterpart, see Section 4.4.3. This yields a deterministic initial line segment $\left(o, L_{1}\right)=\left(o, T\left(F_{0}\right)\right)$, where $L_{1}=(0,1,0)^{\top}$.

Moreover, recall that the random variable $M$, representing the number of parallel fibers, is modeled by a Poisson distribution with parameter $\kappa$. In order to estimate $\kappa$ we first introduce the notion of parallel fibers within a fiber-bundle for the extracted fibers considered in Section 2. Two fibers $f_{1}, f_{2}$ are said to be parallel, i.e., $f_{1} \sim f_{2}$ if it holds that $f_{1} \in f_{2} \oplus b(o, 3 r)$ and $f_{2} \in f_{1} \oplus b(o, 3 r)$. A set of parallel fibers $\left\{f_{1}, \ldots, f_{k}\right\}$ is called a fiber-bundle if for any $i, j \in$ $\{1, \ldots, k\}$ it holds that there exists a path $\left\{i_{1}, \ldots, i_{l}\right\} \subset\{1, \ldots, k\}$ such that

$f_{i} \sim f_{i_{1}}, f_{i_{1}} \sim f_{i_{2}}, \ldots, f_{i_{l}} \sim f_{j}$. Then, we just count the number of parallel fibers per fiber-bundle within the extracted polygonal tracks and get $\kappa=2$ by means of the usual maximum likelihood estimator. Thus, the mean number of fibers in a bundle is given by $\kappa+1=3$.

\subsection{Model Validation}

In the next step the goodness-of-fit of the fiber-bundle model introduced in Equation (4) is investigated.

More precisely, we first define three measures characterizing the curvature of polygonal tracks. Subsequently, these measures are computed for the single 
fibers extracted from the experimental 3D image data of non-woven GDL (given as polygonal tracks) and for the polygonal tracks sampled from the single-fiber model introduced in Section 3.2. Thus, we analyze how adequately the curvatures of extracted and simulated fibers coincide. Note that the simulation of the single fibers is organized as described in [10]. The first curvature measure is defined as follows. Let $p=\left(p_{0}, \ldots, p_{n}\right)$ be a polygonal track given by the starting and end points of its line segments and let $v_{0}(p)=v(p) / d^{2}\left(p_{0}, p_{n}\right)$, where $v(p)$ gives - roughly speaking - the 'area' circumscribed by the polygonal track $p$ and $d\left(p_{0}, p_{n}\right)$ is the length of the line segment $\left(p_{0}, p_{n}\right)$. More precisely, $v(p)$ is given by $v(p)=\int_{p} d\left(x, O\left(\left(x,\left(\left(p_{0}, p_{n}\right)\right)\right) d x\right.\right.$ is the orthogonal projection of the point $x$ located on the polygonal track $p$ to the line segment $\left(p_{0}, p_{n}\right)$.

Moreover, two further curvature characteristics are considered. Namely, the sample variance $S_{z}^{2}(p)$ of the $z$-component of the set of voxel belonging to the discretized polygonal track $p$, representing the movement in $z$-direction of $p$, and some kind of tortuosity $\tau(p)$ of the polygonal track $p$ defined by $\tau(p)=$ $\frac{\sum_{i=0}^{n-1} d\left(p_{i}, p_{i+1}\right)}{d\left(p_{0}, p_{n}\right)}$.

To compare the curvature of single-fibers, these three curvature measures are computed for the extracted and simulated fibers which are drawn from the fitted bundle model. The resulting histograms are displayed in Figure 4, where a very good agreement can be found. This is also indicated by the mean values and standard deviations of these curvature measures listed in Table 1.
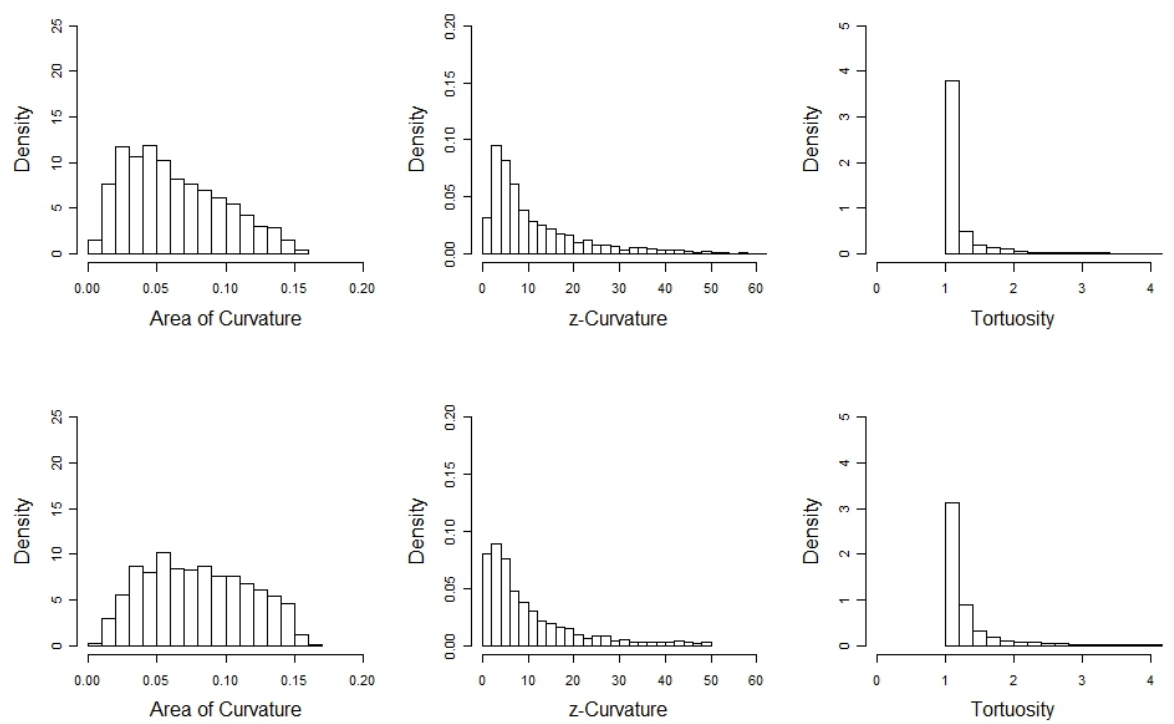

Figure 4: Histograms of $v_{0}(p), S_{z}^{2}(p), \tau(p)$ for extracted (top) and simulated (bottom) tracks 
Table 1: Mean values (standard deviations) of curvature measures $v_{0}(p), S_{z}^{2}(p), \tau(p)$ computed for extracted (top) and simulated (bottom) tracks

\begin{tabular}{llll}
\hline & $v_{0}(p)$ & $S_{z}^{2}(p)$ & $\tau(p)$ \\
\hline extracted fibers & $0.063(0.035)$ & $11.3(10.6)$ & $1.324(1.51)$ \\
simulated fibers & $0.069(0.037)$ & $10.5(9.7)$ & $1.374(1.24)$ \\
\hline
\end{tabular}

\section{Stochastic model for non-woven GDL}

Based on the bundle model introduced in Equation (4), we now develop a stochastic microstructure model of non-woven GDL. First, we consider a system of overlapping fibers generated by a germ-grain model, where the germs form a homogeneous Poisson point process in 3D and the grains are given by the bundle model. Since it is physically impossible that fibers in non-woven GDL overlap mutually, we transform the system of overlapping fibers into a system of spatially regularly distributed, non-overlapping fibers. More precisely, we reduce the size of regions containing no fibers by translating neighboring fibers iteratively towards those regions. Then, this translated system of overlapping fibers is further transformed to a non-overlapping fiber system using an iterative avoidance algorithm. In Section 4.2.2, we show that the changes of curvature of the fibers caused by these transformations are negligible. This iterative avoidance algorithm, based on a force-biased packing approach, was introduced in [1]. It turns out that the model adequately describes the $3 \mathrm{D}$ microstructure of the non-woven GDL considered in this paper, both in terms of structural and physical characteristics. In addition the model has the potential to adequately reproduce the $3 \mathrm{D}$ microstructure of other fiber-based materials as well.

\subsection{Germ-grain model for systems of overlapping fibers}

The construction of the 3D microstructure model for non-woven GDL is motivated by the two-phase superstructure of the experimental GDL data, which is shown in Figure 5. Regarding the cut-out of 3D synchrotron data (Figure 5 , left) we can clearly see that there is a superlattice of horizontally oriented fibers running parallel to the $x$-axis in periodic distances. Thus, we subdivide the microstructure in fiber-channels with some width $h$ where the fibers proceed randomly, and in fiber-bars with some width $b$ where the fibers are mainly running parallel to the $x$-axis. This heterogeneity originates from a production step of the GDL in which fibers are entangled by water jets in order to improve the mechanic stability of the tissue. Thereby, the fiber-bars are not in contact with the water jets which leads to the given superlattice of the GDL. The basic modeling idea is to first consider a uniformly distributed random variable $U$ on the interval $[0, b+h]$ indicating the location of the starting point of the periodic sequence of fiber-channels and fiber-bars. Then, a homogeneous Poisson process $\left\{P_{i}, i \geq 1\right\}$ is generated in $3 \mathrm{D}$ describing the locations of fibers. Subsequently, each point $P_{i}$ is marked either with a bundle of fibers drawn from the bundle 

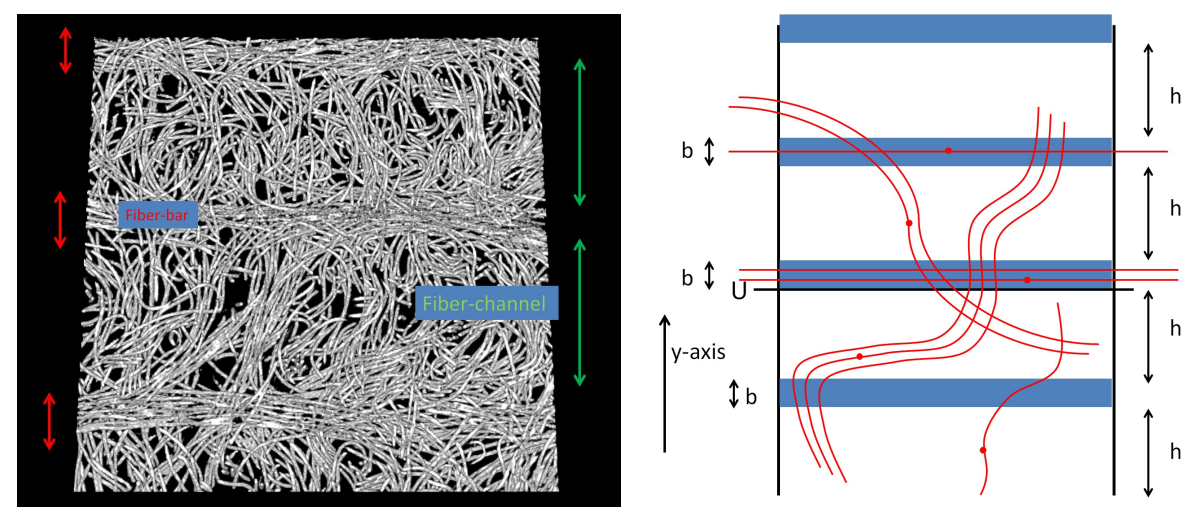

Figure 5: Cut-out of 3D synchrotron data (left), basic idea of bar-channel modeling (right)

model introduced in (4) if $P_{i}$ is located in a fiber-channel, or with line segments parallel to the $x$-axis if $P_{i}$ is located in a fiber-bar. Note that this is an approximation of the experimental fiber morphology within the fiber-bars. However, as can be seen in Section 4.4, the complete model describes the important morphological and physical characteristics sufficiently well. In our approach, the intensity of the homogeneous Poisson process describing the locations of fibers is the same for both the fiber-bar and fiber-channel. If necessary, one could generalize this approach using different intensities within the two regions.

More precisely, the system of overlapping fibers featured with a superlattice is described by a germ-grain model which is given by the set union

$$
\Xi=\bigcup_{i=1}^{\infty}\left(X^{(i)}+P_{i}\right)
$$

where the germs $P_{i}=\left(P_{i 1}, P_{i 2}, P_{i 3}\right)$ form a Poisson point process $\left\{P_{i}\right\}$ in $\mathbb{R}^{3}$ with some intensity $\lambda>0$. The grains $X^{(i)}$ are given by

$$
X^{(i)}= \begin{cases}C^{(i)}, & \text { if } P_{i 2} \in \bigcup_{j \in \mathbb{Z}}[U+j(b+h)-h, U+j(b+h)), \\ B^{(i)}, & \text { else, }\end{cases}
$$

where the $C^{(i)}$ are independent copies drawn from the bundle model $B$ introduced in (4). Furthermore, we define $B^{(i)}$ by

$$
B^{(i)}=\bigcup_{j=0}^{M^{(i)}}\left(B+P_{i}+\delta_{j}^{(i)}\right)
$$

where $B$ is the line segment $B=\left[-(l / 2,0,0)^{\top},(l / 2,0,0)\right]$ and $M^{(i)}$ as well as $\delta_{k}^{(i)}$ are defined like $M$ and $\delta_{k}$ in (4).

Thus, besides the parameters of the bundle model considered in Section 3.2, the model for the system of overlapping fibers has three further parameters $\lambda$, $b$ and $h$ which are specified in Section 4.3. 


\subsection{Iterative avoidance algorithm}

In this section the transformation of the system of overlapping fibers $\Xi$ introduced in (6) to a system of non-overlapping fibers is discussed. Since the fibers of non-woven GDL are extremely regularly distributed in space, i.e., there do not exist large volumes without fibers, we first have to improve the spatial formation of the fibers in $\Xi$ to reduce the amount of vacant volumes in $\Xi$.

\subsubsection{Filling of large "pores"}

We apply an iterative method which translates the fibers hitting the sampling window $W$ towards large 'pores', i.e., towards regions in $W$ with a large distance to the nearest fiber. The basic idea is that the centers of pores attract neighboring fibers. But first of all we introduce the so-called distance transformation $D_{\Xi}$ of $\Xi \cap W$ which assigns each point $x \notin \Xi \cap W$ the shortest Euclidean distance to the nearest fiber. As centers of pores we define all local maxima of $D_{\Xi}$. Let for a given iteration step $c$ be $p_{1}^{(c)}, \ldots, p_{n^{(c)}}^{(c)}$ the centers of pores and let $X_{i_{1}}^{(c)}, \ldots, X_{i_{k}}^{(c)}$ be the random fibers (dilated polygonal tracks) which are not completely located in a fiber-bar and intersect the sampling window $W \subset \mathbb{R}^{3}$. For each pair of pore centers and fibers $\left\{\left(p_{j}^{(c)}, X_{\left(i_{l}\right)}^{(c)}\right): j \in\left\{1, \ldots, n^{(c)}\right\}, l \in\{1, \ldots, k\}\right\}$, we calculate a translation vector $t_{p_{j}^{(c)}, X_{\left(i_{l}\right)}^{(c)}}$ which is given by

$$
t_{p_{j}^{(c)}, X_{\left(i_{l}\right)}^{(c)}}=\mathbb{I}_{\left\{d\left(p_{j}^{(c)}, O_{j, i_{l}}^{(c)}\right)<\frac{3 \max (c)}{2}\right\}} \frac{d\left(p_{j}^{(c)}, O_{j, i_{l}}^{(c)}\right)}{s \max ^{(c)}}\left(O_{j, i_{l}}^{(c)}-p_{j}^{(c)}\right),
$$

where $d(x, y)$ is the Euclidean distance of $x, y \in \mathbb{R}^{3}$ and $O_{j, i_{l}}^{(c)}$ denotes the point located on the surface of the fiber $X_{\left(i_{l}\right)}^{(c)}$ with the shortest Euclidean distance to the pore center $p_{j}^{(c)}$. Moreover, $s$ indicates that $X_{\left(i_{l}\right)}^{(c)}$ is the fiber with the $s$-th shortest distance to $p_{j}^{(c)}$ and $\max ^{(c)}=\max _{j, l} d\left(p_{j}^{(c)}, O_{j, i_{l}}^{(c)}\right)$. The translation vectors given in (7) regulate the attraction of fibers towards pore centers where the attraction is stronger the closer the fiber is located to the pore center and the larger the pore volume is. Based on the translation vectors $t_{p_{j}^{(c)}, X_{\left(i_{l}\right)}^{(c)}}$, we compute a global translation vector $T_{X_{\left(i_{l}\right)}^{(c)}}$ per fiber by

$$
T_{X_{\left(i_{l}\right)}^{(c)}}=\sum_{j=1}^{n^{(c)}} t_{p_{j}^{(c)}, X_{\left(i_{l}\right)}^{(c)}}
$$

In each iteration step, we update the current fiber system replacing $X_{\left(i_{l}\right)}^{(c)}$ by

$$
X_{\left(i_{l}\right)}^{(c+1)}=X_{\left(i_{l}\right)}^{(c)}+\min \left\{T_{\max },\left|T_{X_{\left(i_{l}\right)}^{(c)}}\right|\right\} \frac{T_{X_{\left(i_{l}\right)}^{(c)}}}{\left|T_{X_{\left(i_{l}\right)}^{(c)}}\right|},
$$

where $T_{\max }$ is set to 5 in this paper. After updating the current fiber system, the centers of pores are recalculated and the same translation procedure is repeated. 
Table 2: Mean values (standard deviations) of curvature measures $v_{0}(p), S_{z}^{2}(p), \tau(p)$ computed for simulated fibers before (top) and after (bottom) application of avoidance algorithm

\begin{tabular}{llll}
\hline & $v_{0}(p)$ & $S_{z}^{2}(p)$ & $\tau(p)$ \\
\hline before translation & $0.069(0.037)$ & $10.5(9.7)$ & $1.374(1.24)$ \\
after translation & $0.071(0.041)$ & $10.8(9.8)$ & $1.41(1.35)$ \\
\hline
\end{tabular}

The algorithm stops if $\sum_{l=1}^{k}\left|T_{X_{\left(i_{l}\right)}^{(c)}}\right|<0.002 \sqrt[3]{\nu_{3}(W)}$, where $\nu_{3}(W)$ denotes the volume of $W$. In order to analyze the influence of the pore filling algorithm on the pore size distribution, we computed the mean spherical contact distance for the fiber systems before and after application of the pore filling algorithm. The mean spherical contact distance describes the average distance from a randomly chosen point located in the pore phase to the closest point located in the fiber phase. It turned out that the mean spherical contact distance before applying the pore filling algorithm is equal to 14.6 and after the application of this algorithm it is equal to 6.39. This clearly indicates a large decrease in terms of pore sizes.

\subsubsection{Transformation into systems of non-overlapping fibers}

The fiber system, which is obtained by applying the translation algorithm introduced in Section 4.2.1, is further transformed to a system of non-overlapping fibers. Therefore, we apply the iterative avoidance algorithm introduced in [1]. The principle idea is to first represent each fiber by a chain of spheres where the spheres have radius $r$ and their midpoints are located equidistantly (with small distances from each other) on the center lines of the fibers. Then we apply two different kinds of translations to those spheres: The first translation separates overlapping spheres which belong to different chains of spheres, and the second translation controls the structure of the spheres belonging to the same chain of spheres (and thus the curvature of the fibers). The algorithm terminates when the sum of the norms of all translations falls below a certain threshold. The parameters of the algorithm are chosen as proposed in [1]. The set union $\widetilde{\Xi}$ of the final system of non-overlapping fibers within the sampling window $W$ is denoted by $\widetilde{\Xi} \cap W$.

To show that the changes of fiber structure after applying the avoidance algorithm are minor, we again computed the curvature measures $v_{0}(p), S_{z}^{2}(p), \tau(p)$ for the simulated and subsequently translated fibers. In Table 2, the means and standard deviations of these measures are listed, where we can clearly see that the values are not significantly different.

\subsection{Estimation of model parameters}

It is known from the manufacturer that the width of the fiber-channels is given by $h=500 \mu \mathrm{m}$, and the width of the fiber-bars by $b=70 \mu \mathrm{m}$ for the non-woven GDL material considered in this paper. Furthermore, it is known that the volume fraction of the fiber system is equal to 0.235 . We choose the 
intensity $\lambda$ of the 3D Poisson point process representing the locations of fiberbundles such that the volume fraction of the fiber system $\widetilde{\Xi} \cap W$ coincides with the known volume fraction of 0.235 . Therefore, the minimum-contrast method is used in order to estimate the intensity $\lambda$ of fiber-bundles, i.e.,

$$
\lambda=\operatorname{argmin}_{\lambda}\left|\frac{\nu(\widetilde{\Xi} \cap W)}{\nu_{3}(W)}-0.235\right|,
$$

where we get that $\lambda=1.65 e^{-7}$.

\subsection{Validation of the fitted GDL model}

In Section 4.4.1, we discuss the visual accordance of experimental and simulated data drawn from the GDL model. Subsequently, in Sections 4.4.2-4.4.5, we check more formally if the stochastic 3D model describes the microstructure of the non-woven GDL adequately. In particular, the question arises if this relatively complex model, combining all modeling components, yields a good agreement with the experimental 3D image. Thus, we validate the stochastic microstructure model by comparing physically motivated (transport-relevant) characteristics computed for the experimental non-woven GDL data, and for realizations of the non-woven GDL model. We aim to show that these characteristics which have not been used for model fitting are reflected sufficiently well by our model.

\subsubsection{Visual comparison of experimental and simulated images}

In Figure 6 (right) a realization of the fitted GDL model is shown. The synchroton data in Figure 6 (left) shows clews of fibers in horizontal direction. They are represented in the stochastic model by horizontal fiber bundles as shown in Figure 6 (right). Although, the fiber-bars modeled by straight fiber bundles are a simplification of the microstructure of non-woven GDL, the visual agreement between the simulated and experimental images is quite nice given the complexity of the fiber system.

\subsubsection{Volume fractions within fiber-bar and fiber-channel}

In the following, we analyze the volume fractions of fibers within the fiberbars and the fiber-channels for both experimental fiber systems and fiber systems drawn from the fitted stochastic model. The results given in Table 3 show that the model accurately describes the distribution of fiber volumes.

\subsubsection{Directional distribution of line segments}

In addition to the comparison of experimental and simulated images discussed in Sections 4.4.1-4.4.2, we examine the directional distributions of line segments computed for the extracted polygonal tracks and for the simulated polygonal tracks representing the GDL model introduced in Sections 4.1-4.3. It turns out that both distributions are in an almost perfect accordance to each other, see Figure 7. 

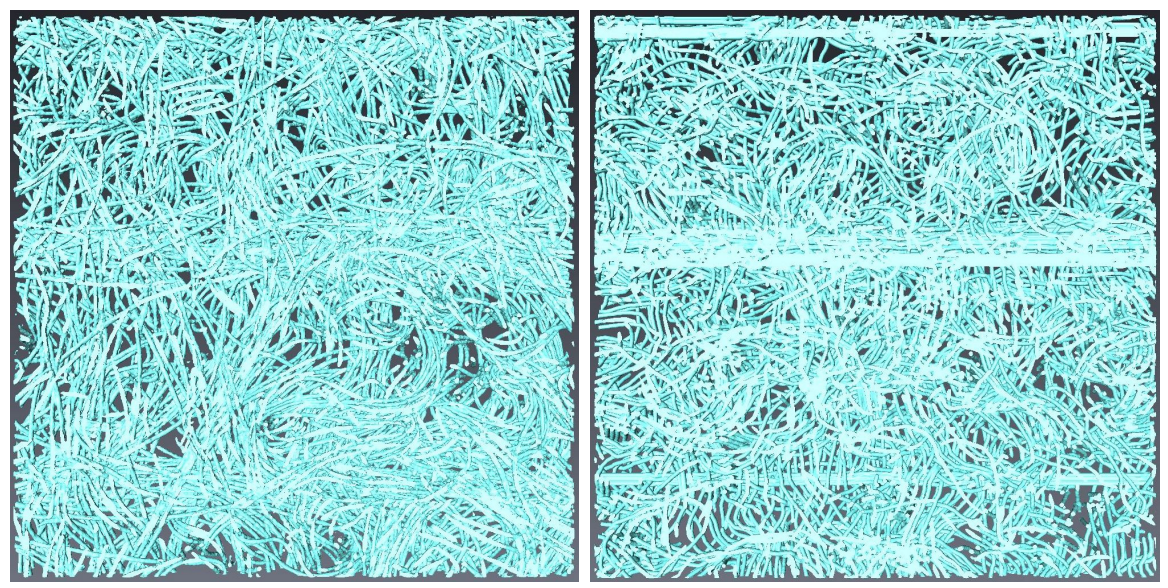

Figure 6: 3D synchrotron data (left) and simulated non-woven GDL (right)

Table 3: Volume fractions computed for fiber-bar and fiber-channel

\begin{tabular}{lll}
\hline & fiber-bar & fiber-channel \\
\hline experimental data & 0.221 & 0.274 \\
simulated data & 0.218 & 0.276 \\
\hline relative error & 0.014 & 0.007 \\
\hline
\end{tabular}
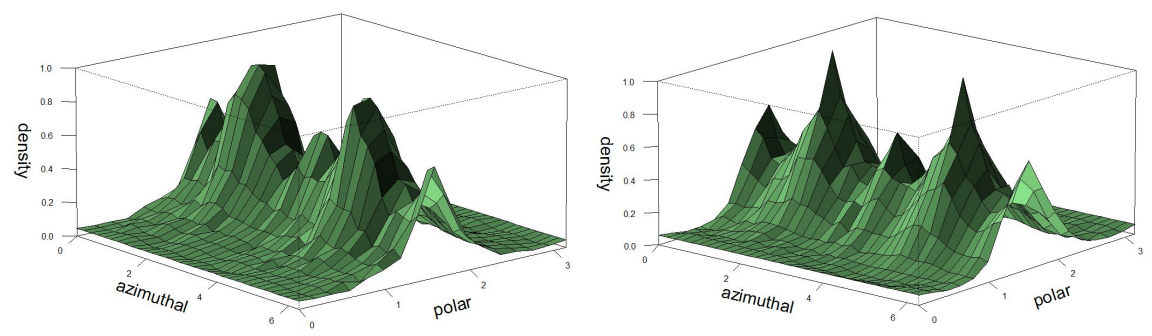

Figure 7: Directional distributions of line segments computed for extracted (left) and simulated (right) polygonal tracks 


\subsubsection{Spherical contact distribution function}

In this section, the spherical contact distribution function $H:[0, \infty] \rightarrow$ $[0,1]$ is computed for experimental and simulated data where $H(t)$ denotes the probability that the minimum distance from a randomly chosen location of the pore phase to the fiber phase $\widetilde{\Xi}$ is not larger than $t$. The results of these

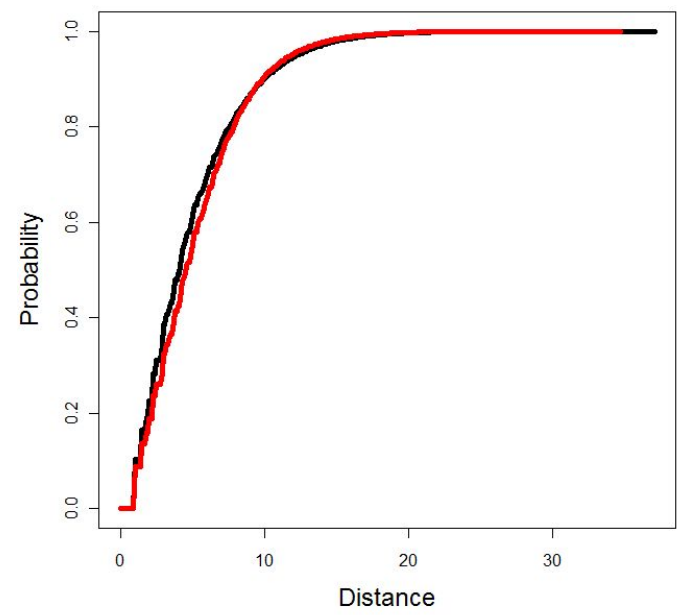

Figure 8: Distribution functions of spherical contact distances for experimental data (black) and simulated data (red) drawn from the fitted GDL model.

calculations are given in Figure 8, where we clearly see that there is a nice accordance between the results obtained for experimental and simulated data. In other words, we see that our model adequately represents this structural characteristic.

\subsubsection{Effective tortuosity}

As another example of a transport-relevant characteristic, we consider the tortuosity of the GDL which describes the lengths of percolating pathways through the pore phase of a porous material, see Figure 9. First recall that the main task of the GDL besides storage and evacuation of water is the gas supply of electrodes. Thus, the geometry of percolating pathways through the pore phase is in a close connection with transportation of gases through the GDL. Note that there exist several definitions of tortuosity. In this paper we define the notion of tortuosity by the ratio of the mean value of flow path lengths through a porous material divided by the thickness of the material.

However, we do not restrict ourselves to the mean value of flow path lengths but consider the histogram of flow path lengths which are calculated based on randomly chosen starting points located at one material's end. This histogram contains much more information about the tortuosity than just a single mean 

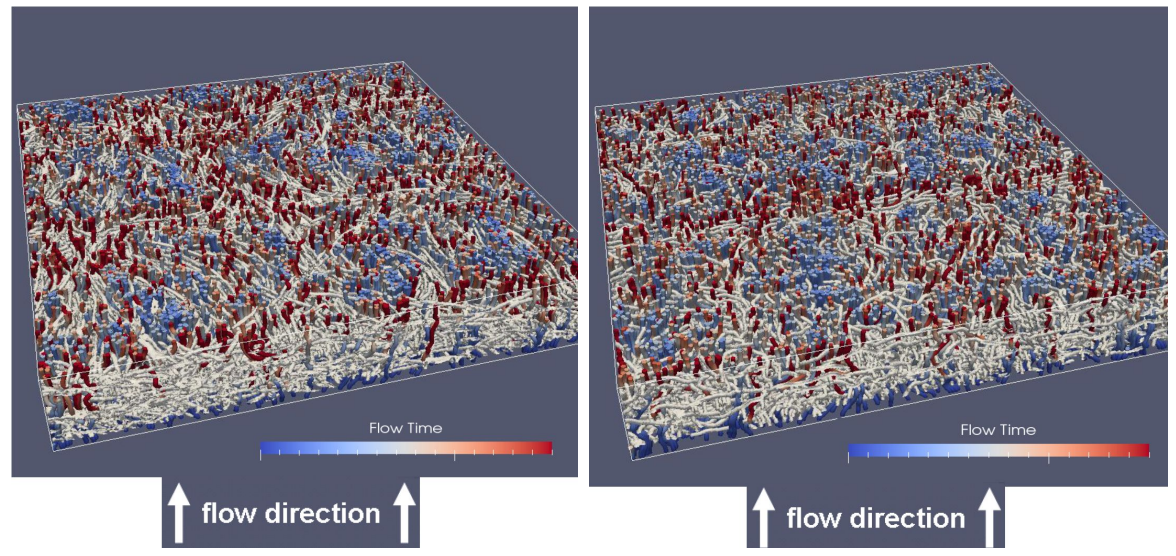

Figure 9: Flow path lines through real (left) and simulated (right) GDL

value.

The computation of flow path lengths can be accomplished via LatticeBoltzman (LB) simulation, see [3, 15]. Notice that the LB-method provides a numerical tool in order to model the transport of fluids under realistic conditions within a given microstructure. Moreover, the LB-method works on 3D binary images with cubic voxel size which are the output of the stochastic model for fiber-based materials introduced in the present paper. Thus, LB-simulations on virtual fiber-based materials generated by stochastic microstructure models can be easily interfaced. For more information about the LB method and the computation of flow path lengths the reader is referred to $[3,15]$.
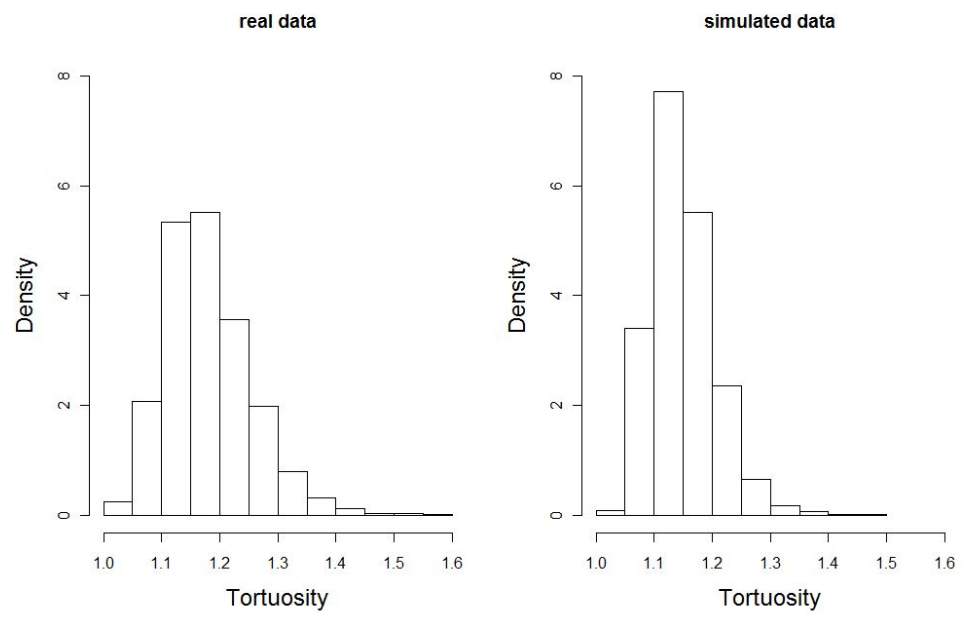

Figure 10: Histograms of flow path lengths for real (left) and simulated data (right) 
Table 4: Means and standard deviations of effective tortuosity computed for experimental and simulated non-woven GDL

\begin{tabular}{lll}
\hline & mean values & standard deviations \\
\hline experimental data & 1.19 & 0.0054 \\
simulated data & 1.15 & 0.0028 \\
\hline relative error & 0.03 & 0.48 \\
\hline
\end{tabular}

The results of these calculations are given in Figure 10, see also Table 4. We clearly see that the results obtained for experimental and simulated data coincide quite nicely since the shapes of the histograms are similar and the two mean values are within the same range, see Table 4 . Thus, altogether, our stochastic GDL model provides an adequate fit to the experimentally measured 3D image of the GDL.

\section{Modeling of PTFE}

GDL are often treated with PTFE to increase hydrophobicity and to reduce blocking of pathways caused by water holdups. Thus, the 3D morphology of PTFE within the GDL has an essential influence on gas transport and water storage, representing important functions for effective operation of PEMFC. The PTFE distribution inside the GDL depends on the preparation procedure as shown in the literature see e.g. [17]. This section deals with the inclusion of PTFE into the non-woven GDL model introduced in Section 4. Unfortunately, 3D image acquisition is still an unsolved challenge, i.e., it is extremely difficult to gain 3D information about the spatial distribution of PTFE. Up to now, 2D microscopic images are the only way to make PTFE visible within the GDL. In Figure 11, two 2D SEM images of non-woven GDL are shown, containing different amounts of PTFE (measured in weight percentage), where the PTFE accumulates in areas between neighboring fibers. Moreover, by means of normalized X-ray signals gained by BEC-images from cross-sections of non-woven GDL, it is possible to approximate the volume fraction of PTFE in thin slices of the GDL, where three different total amounts (10\%, $20 \%$ and $40 \%)$ of PTFE have been considered. This is done by separating the PTFE, fibers and pores in the $2 \mathrm{D}$ cross-section images and subsequently analyzing the volume fractions in dependency of the location within the GDL.

It turned out that in all three cases the PTFE is not uniformly distributed within the GDL. More precisely, the PTFE concentrates in the deeper and upper zones of the GDL, whereas in the middle part there is hardly any PTFE, see Figure 12.

Since we are interested in the volume fractions of PTFE in thin slices for any percentage-value $y$ of PTFE, say for all $y \in[0,50]$, we fit a function $s$ : $[0,200] \times[0,50] \rightarrow[0,1]$ to the three available functions of volume fraction in thin slices using non-linear regression, see Figure 13. The resulting volume 


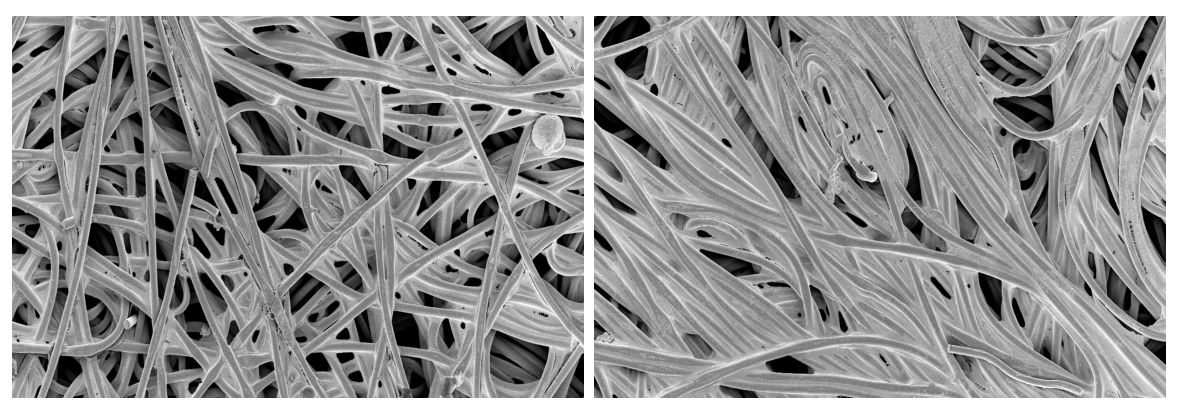

Figure 11: 2D SEM images of non-woven GDL containing 10\% (left) and 20\% (right) PTFE.

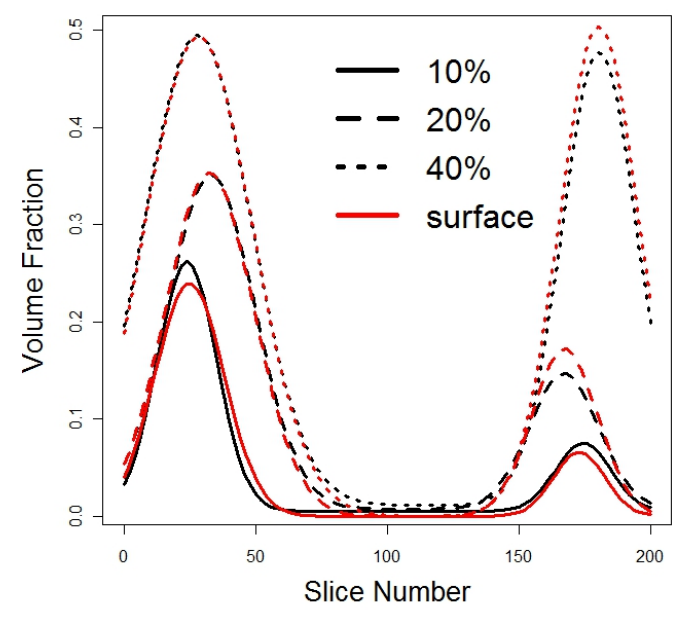

Figure 12: Volume fractions of PTFE in thin slices of GDL (black lines) and the corresponding approximations by the function $s(\cdot, \cdot)$ (red lines).

fractions $s(x, y)$ of PTFE can then be given in a parametrized form, where

$$
\begin{aligned}
s(x, y)= & \left(0.04 \log (y+1)+0.025 \log (y+1)^{2}\right) \\
& \cdot \exp \left(-\left(\frac{x+1.96 y-7.74 \log (y+1)^{2}}{7.77 \log (y+1)}\right)^{2}\right) \\
& +\left(0.0046 y+0.0002 y^{2}\right) \\
& \cdot \exp \left(-\left(\frac{200-x+2.83 y-9.62 \log (y+1)^{2}}{5.76 \log (y+1)}\right)^{2}\right) .
\end{aligned}
$$

In this formula, $x$ denotes the distance to the first slice of the GDL and $y$ denotes 


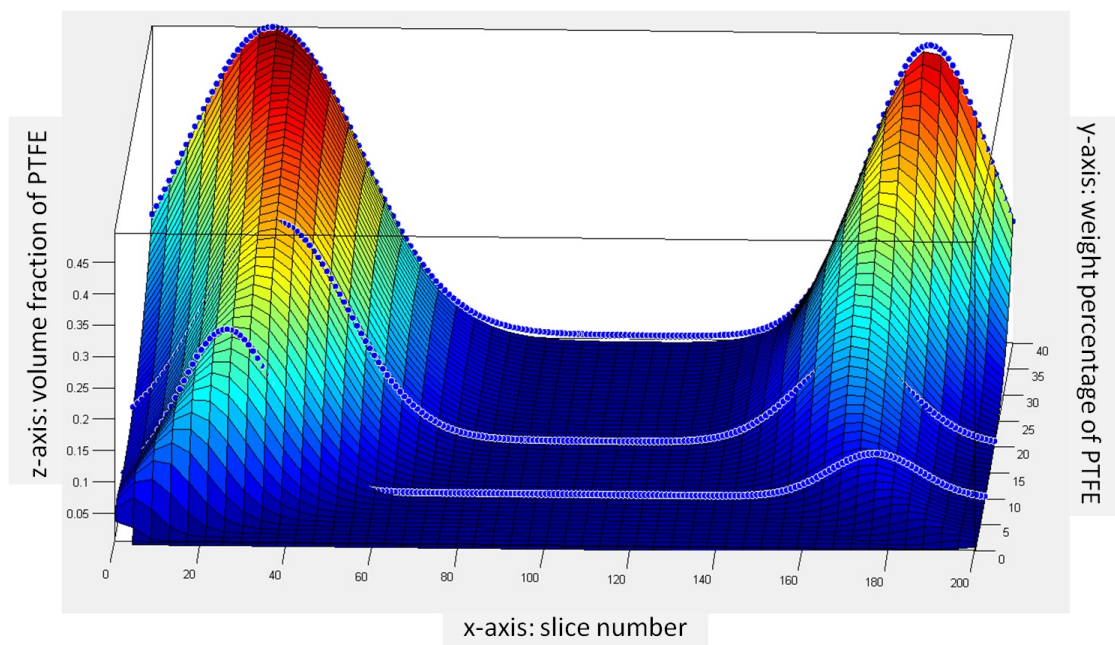

Figure 13: Volume fractions of PTFE in thin slices for total amounts of PTFE (measured in weight percentage) between 0 and 50 .

the weight percentage of PTFE, i.e., $s(x, y)$ is the volume fraction of PTFE in the $x$-th slice of the GDL while altogether $y$ weight percentage of PTFE is included. Thus, we are now able to forecast the volume fractions of PTFE in thin slices for all (total) weight percentages of PTFE between 0 and 50 .

Our approach to including PTFE into the GDL model $\widetilde{\Xi}$ of non-overlapping fibers, introduced in Section 4, is to take into account that the PTFE accumulates in areas between neighboring fibers and that the volume fraction $s(x, y)$ of PTFE in thin slices depends on the distance $x$ to the surface of the GDL and on the total amount $y$ of PTFE. Therefore, we define a function $D_{\Xi}: \mathbb{R}^{3} \rightarrow \mathbb{R}$ where $D_{\widetilde{\Xi}}(v)$ is the sum of the shortest distances $d_{1}(v)$ and $d_{2}(v)$ from location $v \in \mathbb{R}^{3} \backslash \widetilde{\Xi}$ to its two nearest fibers, i.e., we put

$$
D_{\Im}(v)= \begin{cases}\infty, & \text { if } v \in \widetilde{\Xi}, \\ d_{1}(v)+d_{2}(v), & \text { else. }\end{cases}
$$

Then, for each slice $x \in[0,200]$ and for each PTFE weight percentage $y \in[0,50]$, we allocate those voxel to PTFE for which the value of $D_{\widetilde{\Xi}}$ is smaller than a certain threshold $t_{x y}$, where the threshold $t_{x y}$ is chosen such that the resulting volume fraction of PTFE in the $x$-th slice fits the predicted volume fraction $s(x, y)$, see Figure 14. Note that each slice has a thickness of $1 \mu m$.

\section{Conclusions}

A novel, realistic stochastic 3D model has been introduced which describes the microstructure of non-woven GDL consisting of strongly curved, non-over- 


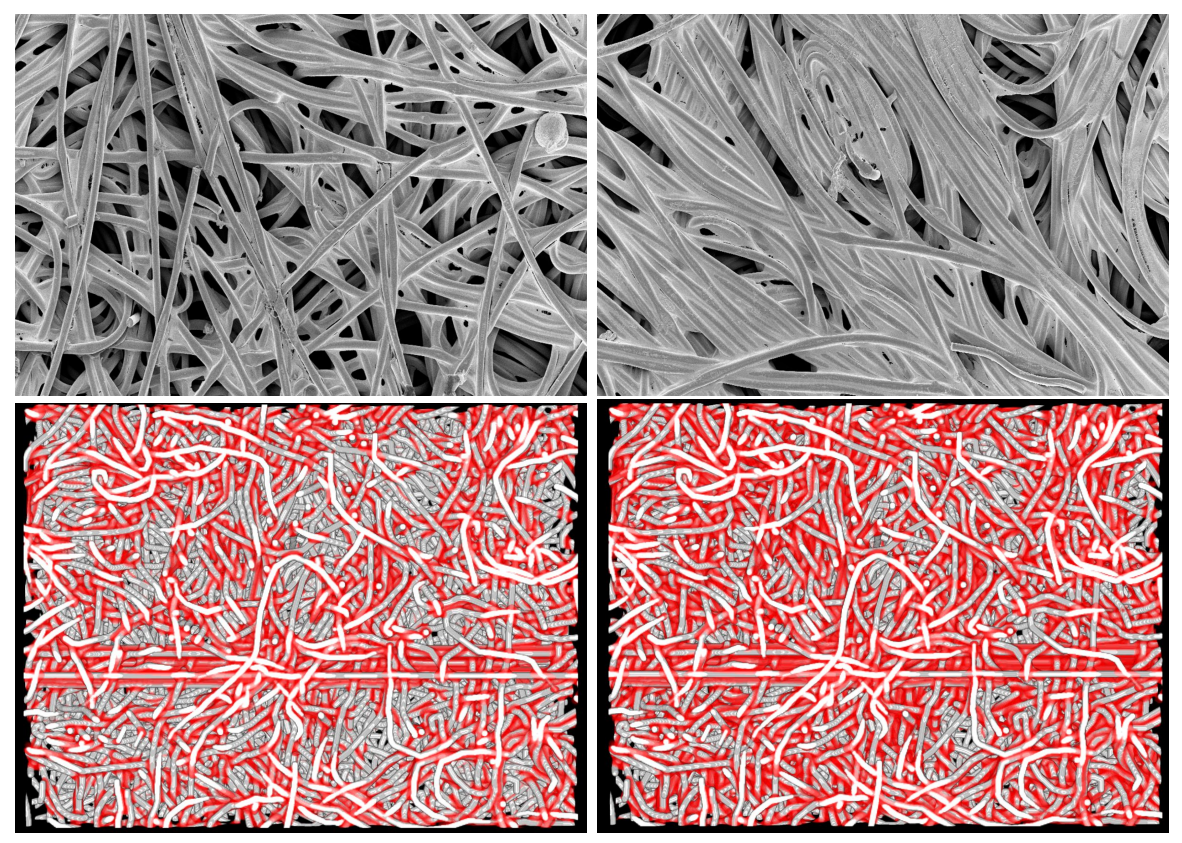

Figure 14: 2D SEM images of non-woven GDL (top) and simulated non-woven GDL (bottom) containing $10 \%$ (left) and 20\% (right) PTFE.

lapping fibers. The model is constructed in a two-stage approach: In the first stage, a system of random fibers is generated by random 3D polygonal tracks using multivariate time series. In the second stage, this system of fibers is transformed into a system of non-overlapping fibers. The parameters of this model have been fitted to 3D image data of non-woven GDL. Subsequently, the model has been validated by comparing transport-relevant characteristics computed for experimental and virtually generated non-woven GDL data. A good agreement was found. Moreover, we included PTFE into our GDL model, whose 3D morphology is of great importance in order to study the correlation between the structure of a GDL and its functionality.

\section{Acknowledgements}

This research has been supported by the German Federal Ministry of Education and Research (BMBF) in the framework of the priority program 'Mathematics for Innovations in Industry and Services'.

\section{References}

[1] Altendorf H, Jeulin D. Random walk based stochastic modeling of 3D fiber systems. Phys Rev E 2011; 83: 041804. 
[2] Bezrukov A, Stoyan D. Simulation and statistical analysis of random packings of ellipsoids. Part Part Syst Char 2006; 23: 388-398.

[3] Brinkmann JP, Froning D, Reimer U, Schmidt V, Lehnert W, Stolten D. 3D modeling of one and two component gas flow in fibrous microstructures in fuel cells by using the Lattice-Boltzmann method. ECS Transactions 2012; 50(2): 207-219.

[4] Coelho D, Thovert JF, Adler P. Geometrical and transport properties of random packings of spheres and aspherical particles. Phys Rev E 1997; 55: 1959-1978.

[5] Daino MM, Kandlikar SG. 3D phase-differentiated GDL microstructure generation with binder and PTFE distributions. Int J Hydrogen Energy 2012; 37: 5180-5189.

[6] Feder J. Random sequential adsorption. J Theor Biol 1980; 87: 237-254.

[7] Fuller WA. Introduction to statistical time series. 2nd ed. New York: J. Wiley \& Sons; 1996.

[8] Gaiselmann G, Kulik R, Schmidt V. Statistical inference for curved fibrous objects in 3D, based on multiple observations of short multivariate autoregression processes. Comput Stat Data Anal 2013; (submitted).

[9] Gaiselmann G, Manke I, Lehnert W, Schmidt V. Extraction of curved fibers from 3D data. Image Anal Stereol 2013; 32: 57-63.

[10] Gaiselmann G, Thiedmann R, Manke I, Lehnert W, Schmidt V. Stochastic 3D modeling of fiber-based materials. Comp Mater Sci 2012; 59: 75-86.

[11] Hartnig C, Jörissen L, Kerres J, Lehnert W, Scholta J. Polymer electrolyte membrane fuel cells (PEMFC). In: Gasik M. editor. Materials for fuel cells, Cambridge: Woodhead Publishing; 2008, p. 101-184.

[12] Illian J, Penttinen A, Stoyan H, Stoyan D. Statistical analysis and modelling of spatial point patterns. Chichester: J. Wiley \& Sons; 2008.

[13] Inoue G, Yoshimoto T, Matsukuma Y, Minemoto M. Development of simulated gas diffusion layer of polymer electrolyte fuel cells and evaluation of its structure. J Power Sources 2008; 175: 145-158.

[14] Jia K, Li X. Water transport in polymer electrolyte membrane fuel cells. Prog Energy Combust Sci 2011; 37: 221-291.

[15] Koponen A, Kataja M, Timonen J. Tortuous flow in porous media. Phys Rev E 1996; 54(1): 406-410.

[16] Lütkepohl H. New introduction to multiple time series analysis. Berlin: Springer; 2006. 
[17] Mathias MF, Roth J, Fleming J, Lehnert W. Diffusion media materials and characterisation. In: Vielstich W, Lamm A, Gasteiger H, editors. Handbook of fuel cells, London: J. Wiley \& Sons; 2003, p. 517-537.

[18] Ohser J, Schladitz K. 3D images of materials structures - processing and analysis. Weinheim: Wiley-VCH; 2009.

[19] Provatas N, Haataja M, Asikainen J, Majaniemi S, Alava M, Ala-Nissila T. Fiber deposition models in two and three spatial dimensions. Colloids Surf, A 2000; 165: 209-229.

[20] Schulz VP, Becker J, Wiegmann A, Mukherjee PP, Wang CY. Modeling of two-phase behavior in the gas diffusion medium of PEFCs via full morphology approach. J Electrochem Soc 2007; 154: B419-B426.

[21] Stoyan D, Kendall WS, Mecke J. Stochastic geometry and its applications. 2nd ed. Chichester: J. Wiley \& Sons; 1995.

[22] Thiedmann R, Fleischer F, Lehnert W, Schmidt V. Stochastic 3D modelling of the GDL structure in PEM fuel cells, based on thin section detection. J Electrochem Soc 2008; 155: B391-B399.

[23] Widom B. Random sequential addition of hard spheres to a volume. J Chem Phys 1966; 44: 3888-3894.

[24] Williams SR, Philipse AP. Random packings of spheres and spherocylinders simulated by mechanical contraction. Phys Rev E 2003; 67: 051301. 